\section{Efeitos do método isostretching sobre parâmetros morfológicos e sobre um conjunto de testes motores em idosas}

\section{Effects of the isostretching method on morphological parameters and on a set of motor tests in the elderly}

\author{
Christina Cruz Cepeda ${ }^{1,2}$ \\ André Luiz Félix Rodacki ${ }^{2}$ \\ Leslie Nathan Persch ${ }^{1}$ \\ Peterson Pereira Silva \\ Silvia Buba ${ }^{1}$ \\ Vanessa Freitas Dressler ${ }^{1}$
}

Resumo - O treinamento resistido de intensidade leve e moderada parece eficaz em prover importantes melhorias sobre a força, o equilíbrio e a funcionalidade em idosos. O objetivo do presente estudo foi analisar os efeitos de 12 semanas de treinamento do Método Isostretching sobre a capacidade funcional e arquitetura muscular em idosas. A amostra foi de 25 voluntárias ( $n=25)$, divididas em um grupo controle (GC; $n=11 ; 62,3$ $\pm 1,9$ anos; $1,58 \pm 0,06 \mathrm{~m} ; 73,4 \pm 1,4 \mathrm{~kg}$ ) que prosseguiu suas atividades físicas habituais e um grupo experimental (GE; $\mathrm{n}=14 ; 64,2 \pm 4$,3anos; $1,56 \pm 0,05 \mathrm{~m} ; 74,2 \pm 1,6 \mathrm{~kg}$ ) que realizou um programa de exercícios de isostretching. As avaliações compreenderam $\mathrm{Ti}$ med Up and Go -TUG, avaliação de Tinetti e avaliação dos parâmetros morfológicos por meio da Ultrassonagrafia. Os resultados mostraram que o GE apresentou uma melhora $(\mathrm{p}<0,05)$ tanto no TUG quanto no Tinetti. Nos parâmetros morfológicos do vasto lateral no grupo GE observou-se: aumento médio de $3.6 \mathrm{~mm}$ do comprimento do fascículo em relaxamento ( $\mathrm{p}<0,05 ; \mathrm{ES}=1,15)$, em contração isométrica aumento de $4,9 \mathrm{~mm}(\mathrm{p}<0.05$; $\mathrm{ES}=1,94)$; no ângulo de penação na condição relaxada, um aumento de $16 \%(\mathrm{p}<0.05$; $\mathrm{ES}=0,70)$, em contração isométrica, aumento de $12 \%(\mathrm{p}<0,05 ; \mathrm{ES}=0,50)$; já na espessura $(\mathrm{p}<0,05 ; \mathrm{ES}=0,52)$ aumento de $8 \%$ relaxado, em contração isométrica $(\mathrm{p}<0,05 ; \mathrm{ES}=0,43)$ de $9 \%$. O GC não apresentou alterações significativas. O treinamento isométrico por meio do isostretching promoveu alterações nos parâmetros morfológicos musculares e melhorou as capacidades funcionais em idosas.

Palavras-chave: Adaptação muscular; Equilíbrio; Envelhecimento; Treinamento de resistência.

\footnotetext{
Abstract - Moderate intensity resistance training seems to be effective in providing significant improvements on strength, balance and function in the elderly. This study aimed to analyze the effect of a 12-week isostretching training on functional capacity and muscle architecture in elderly women. The sample included 25 volunteers $(n=25)$ divided into a control group $(C G, n=11,62.3 \pm 1.9$ years old, $1.58 \pm 0.06 \mathrm{~m}, 73.4 \pm 1.4 \mathrm{~kg}$ ) that followed their physical activity habits and an experimental group ( $E G, n=14,64.2 \pm 4.3$ years, $1.56 \pm 0.05 \mathrm{~m}, 74.2 \pm$ $1.6 \mathrm{~kg}$ ) that was submitted to an isostretching program. The following assessments were used: Timed Up and Go-TUGT, Tinetti balance test and evaluation of morphological parameters through ultrasound imaging technique. The results showed that the EG improved $(p<0.05)$ in both TUGT and Tinetti. In the morphologic parameters of the vastus lateralis muscle in the EG, we observed the following: mean increase of $3.6 \mathrm{~mm}$ in length issue relaxation ( $p$ $<0.05, E S=1.15)$; increase of $4.9 \mathrm{~mm}$ in isometric contraction $(p<0.05 ; E S=1.94)$; increase of $16 \%$ in the pennation angle in the relaxed condition $(p<0.05 ; E S=0.70)$; increase of $12 \%$ in isometric contraction ( $p<0.05 ; E S=0.50)$; increase of $8 \%$ in thickness in the relaxed condition $(p<0.05, E F=0.52)$; and increase of $9 \%$ in isometric contraction $(p<0.05, E F$ $=0.43$ ). The GC did not show any significant changes. The isometric training through the isostretching method promoted changes in the morphological and muscle parameters and also improved the functional abilities in elderly women.
}

Key words: Aging; Balance; Muscle adaptation; Resistance training.
1Universidade Positivo. Curso de Fisioterapia. Curitiba, PR. Brasil.

2Universidade Federal do Paraná. Programa de Pós Graduação em Educação Física. Curitiba. PR. Brasil.

Recebido em 19/11/12 Revisado em 22/01/13 Aprovado em 22/02/13 


\section{INTRODUÇÃOO}

O processo de envelhecimento leva a uma série de alterações fisiológicas sobre os sistemas nervoso, musculoesquelético e sensorial ${ }^{1}$. As alterações mais frequentes causam reduções da massa e força muscular ${ }^{2-4}$.Tais alterações interferem na capacidade contrátil e se refletem sobre a funcionalidade, além de causarem aumento sobre o risco de quedas em idosos ${ }^{4,5}$.

Com o envelhecimento, a diminuição da massa muscular e as mudanças na arquitetura muscular causam pronunciada perda sobre a capacidade de produção de força, especialmente, após a terceira década de vida ${ }^{6,7}$. As principais mudanças na arquitetura muscular decorrentes do envelhecimento envolvem alterações na espessura, no comprimento do fascículo e no ângulo de penação ${ }^{6,8,9}$. O comprimento do fascículo está relacionado com a velocidade de contração muscular enquanto o ângulo de penação está associado com a capacidade de produzir elevados níveis de força ${ }^{9-12}$.

O treinamento de resistência é considerado como um método eficaz para reverter o processo de redução da força e das adaptações morfológicas do músculo esquelético associadas com o aumento da idade. Apesar dos benefícios do treinamento de alta intensidade na capacidade de produzir força e num conjunto de aspectos morfológicos musculares, melhorias sobre o desempenho funcional nem sempre têm sido observadas. Por outro lado, alguns estudos que envolveram treinamento com cargas de intensidade leve e moderada também têm sido demonstrados como eficazes em prover importantes melhorias sobre a força e o equilíbrio, as quais também têm promovido melhorias da funcionalidade em idosos ${ }^{13-15}$.

Interessantemente, alguns estudos têm reportado aumentos similares na força em idosos, independente da intensidade do treinamento resistido. Pruitt et al. ${ }^{16}$, observaram aumentos de $45 \%$ na força após um período de treinamento resistido realizado com intensidade de 80\% de 1 Repetição Máxima (RM) e 42\% quando intensidades consideravelmente menores foram aplicadas ( $40 \%$ 1RM). Hortobagyi et al. ${ }^{17}$ encontraram ganhos similares na força máxima (29\%) após treinamento de baixa (40\% 1 RM) e alta intensidade ( $80 \% 1 \mathrm{RM})$. Estudos que envolveram contrações isométricas também têm demonstrado ganhos na força muscular. Kubiak et al. ${ }^{18}$ observaram aumentos de $33 \%$ na força isométrica do quadríceps após treinamento isométrico realizado com intensidade de $45 \%$ da contração isométrica voluntária máxima (CIVM).

O treinamento de alta resistência tem resultado em importantes modificações da arquitetura muscular ${ }^{19}$, no entanto, programas de treinamento com intensidade menores (leve e moderada) também têm sido reportados como efetivos para causarem alterações na arquitetura muscular. Lee ${ }^{20}$ aplicou cargas de $50 \%$ da capacidade máxima por meio de eletroestimulação, que resultou em aumento do ângulo de penação de $4^{\circ}$ e ganhos de $26 \%$ na força máxima. Alegre et al. ${ }^{9}$ também reportaram aumentos de 6,9\% na espessura do vasto lateral e de $10,3 \%$ no comprimento dos fascículos em resposta a um programa resistido de intensidade leve. Dessa forma, 
observa-se que o treinamento isométrico com intensidade leve e moderada pode causar modificações importantes sobre a função contrátil (ex. força máxima) e sobre parâmetros morfológicos do tecido muscular (ex. espessura, ângulo de penação e comprimento do fascículo).

O Isostretching é um método composto por exercícios que envolvem contrações isométricas de intensidade leve e moderada, em que exercícios generalizados são realizados em posições estáticas que visam preservar as curvas fisiológicas da coluna vertebral. $\mathrm{O}$ método tem por objetivos promover o fortalecimento muscular, aumentar a flexibilidade e corrigir e/ou melhorar a postura, além de aprimorar o controle neuromuscular, melhorar a consciência corporal e a capacidade respiratória. Alguns estudos têm demonstrado os benefícios do Isostretching sobre alterações posturais, quadros álgicos da coluna vertebral, capacidade respiratória e, mais recentemente, na capacidade funcional de idosos ${ }^{21,22}$.

Sanglard et al. ${ }^{21}$, observou que idosos submetidos a 24 sessões de um programa de exercícios de isostretching apresentaram melhor controle postural, desempenho de atividades de vida diária e diminuição da probabilidade de quedas quando avaliados por meio do Teste de Alcance Funcional, Escala de Equilíbrio de Berg, Avaliação de Tinetti e o Teste de Romberg e Romberg-Barré. Carvalho e Assini ${ }^{22}$ também reportaram melhorias sobre a capacidade funcional de idosas por meio do Teste de Caminhada de 6 minutos após 10 sessões de treinamento.

Sendo assim, existem indícios de que o método isostretching pode causar melhorias sobre parâmetros morfológicos do tecido muscular e na capacidade funcional de idosos. Este tipo de treinamento pode constituir uma forma atrativa de treinamento, visto que exercícios leves e moderados tendem a ser menos lesivos em idosos, e as degenerações decorrentes da idade podem ser agravadas pelas elevadas cargas aplicadas no treinamento de alta intensidade. Portanto, este estudo analisou os efeitos de um programa de treinamento envolvendo 12 semanas (36 sessões) do Método Isostretching sobre o equilíbrio/agilidade, marcha e arquitetura muscular de idosas saudáveis.

\section{PROCEDIMENTOS METODOLÓGICOS}

A amostra por conveniência foi composta por 25 idosas com idade mínima de 60 anos, que estavam engajadas nos programas de atividade física há mais de seis meses, promovidos pela Secretaria Municipal de Esporte e Lazer em Curitiba-PR. Idosas em tratamento para lombalgia, que utilizassem dispositivos para deambulação, história de fratura recente, hipertensão não controlada e uso contínuo de medicamentos que pudessem interferir no estudo, não foram incluídas.

As idosas receberam informações sobre os procedimentos experimentais e assinaram um termo de consentimento livre e esclarecido, o qual foi aprovado pelo Comitê de Ética e Pesquisa da Universidade Positivo sob n ${ }^{\circ}$ 131.10, estando de acordo com a Resolução196 de 10/10/1996 do Conselho Nacional de Saúde (CNS). 
O nível de atividade física foi determinado pelo Questionário Internacional de Atividade Física (IPAQ) ${ }^{23}$, que identificou todas as participantes como ativas. As voluntárias $(\mathrm{n}=25)$ foram distribuídas de forma balanceada aleatória, em um grupo controle (GC; $\mathrm{n}=11 ; 62.3 \pm 1,9$ anos; $1,58 \pm 0,06 \mathrm{~m}$; $73,4 \pm 1,4 \mathrm{~kg}$ ) que deu continuidade ao programa de atividade física que realizava antes do início do experimento (exercícios de ginástica localizada de baixa intensidade com duração de 50 minutos e periodicidade de três sessões semanais). e um grupo experimental (GE; $n=14 ; 64,2 \pm 4$,3anos; $1,56 \pm 0,05 \mathrm{~m} ; 74,2 \pm 1,6 \mathrm{~kg}$ ) que foi submetido a um programa de exercícios de isostretching. O GE teve três sessões semanais durante doze semanas ininterruptas, as quais foram ministradas pela mesma pesquisadora e tiveram frequência mínima de $75 \%$. Os exercícios realizados pelo GC permaneceram inalterados durante o período do experimento e tiveram uma frequência mínima superior a $85 \%$. As participantes foram avaliadas em duas sessões idênticas que foram realizadas antes (PRE) e após (POS) o período de intervenção.

A duração de cada sessão de isostretching foi de 50 minutos, os quais compreenderam 15 minutos de aquecimento (exercícios aeróbicos de intensidade leve a moderada), 30 minutos de exercícios baseados no método isostretching e 5 minutos finais (exercícios de relaxamento).

Em cada aula, foram escolhidas de seis a sete posturas, em decúbito dorsal (quadril em flexão de $90^{\circ}$, extensão de joelhos, flexão de tornozelo, flexão de ombro, extensão de cotovelo e punho), sentado (coluna vertebral alinhada, quadril em flexão de $90^{\circ}$, joelhos em flexão e ou extensão, tornozelos em flexão e membros superiores em amplitudes articulares diversas) e na posição ortostática. A contração simultânea dos músculos extensores da coluna, abdominais e glúteos foi mantida durante a postura, os membros inferiores e superiores posicionados, respeitando a amplitude articular individual e mantida durante o período expiratório que durou no mínimo 5 s e repetida três vezes. Um intervalo de $15 \mathrm{~s}$ foi imposto entre cada tentativa. O grau de dificuldade foi incrementado, após a décima sessão, com uso de bastões e bolas de borracha.

\section{Questionário Internacional de Atividade Física (IPAQ)}

Foi utilizada a versão 8, forma longa, semanal usual adaptado para idosos, que apresenta 27 questões e permite estimar o tempo semanal gasto em atividades físicas de intensidade leve, moderada e vigorosa, em diferentes contextos do cotidiano ${ }^{23}$.

\section{Timed "UP AND GO" Test (TUG)}

O TUG consiste na mensuração do tempo necessário para percorrer 3 metros, partindo da posição sentada, circundar um cone e retornar a mesma posição sentada. Para efeitos do presente estudo, tempos superiores a 9s foram considerados como indicadores para risco de quedas para esta faixa etária ${ }^{24}$. 


\section{Instrumento de Avaliação de TINETTI}

O teste é composto por duas partes. A primeira avalia nove aspectos do equilíbrio com pontuação entre 0 a 16, enquanto a segunda avalia os sete aspectos da marcha com pontuação variando de 0 a 12, totalizando 28 pontos. Pontuação menor que 19 pontos é considerada como alto risco de quedas, entre 19 e 24 pontos como de risco moderado e acima de 25 pontos com baixo risco ${ }^{2}$.

\section{Parâmetros Morfológicos - Ultrassonografia}

A avaliação da arquitetura muscular do músculo vasto lateral direito, e as medidas foram realizadas por ultrassonografia (General Eletric ${ }^{\oplus}$, modelo Logiq Book XP), com transdutor multifrequencial de $11 \mathrm{MHz}$ de alta resolução.

A participante foi posicionada em decúbito dorsal, com os membros inferiores estendidos e com os joelhos flexionados a $10^{\circ}$. A posição do joelho controlada por um goniômetro manual (Carci甲) e mantida ao longo do teste com o auxílio de um rolo de espuma posicionado na linha da fossa poplítea. Tal posição foi adotada por permitir maior reprodutibilidade das medidas ${ }^{25}$. As medidas foram tomadas no terço médio da coxa, tomando-se o trocânter maior do fêmur e linha articular do joelho como referência. Para garantir que as medidas pudessem ser realizadas da mesma forma, uma fita adesiva guia com $0,5 \mathrm{~cm}$ de largura e $10.0 \mathrm{~cm}$ de comprimento foi fixada longitudinalmente à pele. Perpendicularmente à fita adesiva longitudinal, um conjunto de 4 fitas de $0,3 \mathrm{~cm}$ de largura e $3,0 \mathrm{~cm}$ de comprimento foram fixadas a fim de permitir o agrupamento das imagens a cada tomada ao longo do músculo ${ }^{12}$.

As imagens foram coletadas com um transdutor retangular de $5 \mathrm{~cm}$ de comprimento por $2 \mathrm{~cm}$ de largura, posicionado perpendicularmente à superfície do ventre muscular, sendo a imagem no sentido longitudinal das fibras musculares. Foram coletadas quatro imagens do músculo em repouso e quatro em contração isométrica submáxima. As sombras deixadas pela fita adesiva permitiram reagrupar as imagens em um programa de gerenciamento de imagens (Corel Draw X $5^{\circ}$ ).

A espessura foi definida como a distância entre aponeurose superior e inferior profunda do músculo 9 . O ângulo de penação foi definido como o ângulo entre a direção das fibras musculares e a linha de geração de força de um músculo, enquanto o comprimento do fascículo foi definido como o comprimento da linha fascicular entre as inserções do mesmo na aponeurose inferior e superior profunda ${ }^{12,24,25}$.

\section{Procedimentos Estatísticos}

Inicialmente, a normalidade dos dados foi verificada através do teste de Shapiro-Wilk, enquanto a homogeneidade foi determinada por intermédio do teste de Levene. Diferenças intra e intergrupos em variáveis que tiveram sua normalidade confirmada foram verificadas através de um conjunto de ANOVAs para dois fatores (tempo [PRE vs POS] e grupo [GE vs GC]). O teste post-hoc de Scheffé foi aplicado para determinar onde as diferenças 
encontradas na análise de variância ocorreram (tempo e grupo). Os dados que não tiveram sua normalidade confirmada foram analisados com o teste de Fisher. O tamanho do efeito (ES) foi quantificado pelo teste de Cohen's. O nível de significância estatística considerado foi de $\mathrm{p}<0,05$ e os dados foram analisados pelo programa STATISTICA 7.

\section{RESULTADOS}

\section{Time up and Go Test - TUG}

Os grupos não apresentaram diferenças antes do programa de intervenção (PRE; $>0,05 ; E S=0,36$ ). Após o período de intervenção (POS),o grupo experimental apresentou uma redução $(\mathrm{p}<0,05 ; \mathrm{ES}=0,85)$ de $11 \%$ no desempenho do teste, enquanto o grupo controle apresentou uma redução de $4 \%$ (p>0,05; ES=0,36) após o mesmo período de tempo (FIGURA 1).

TUG
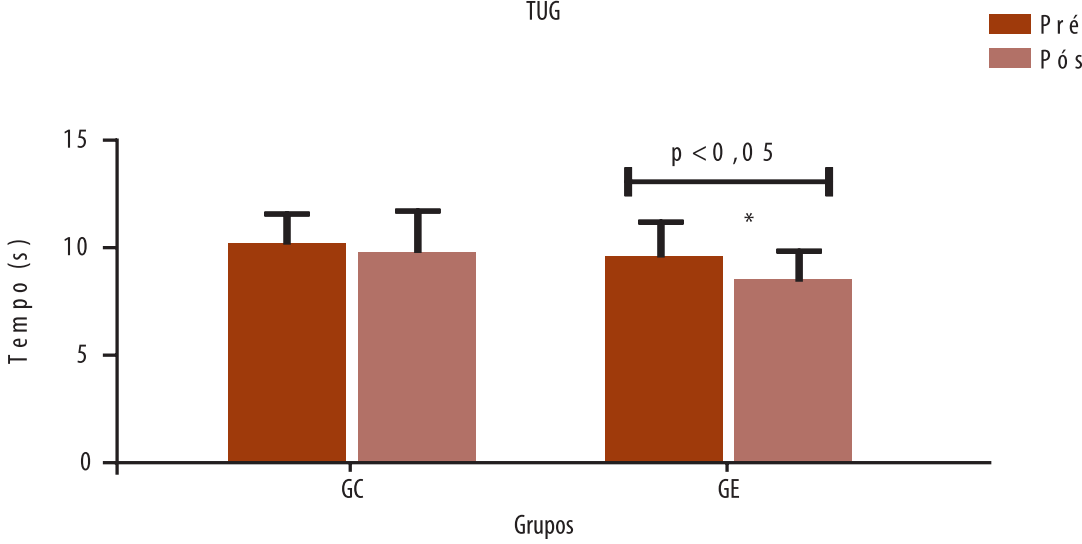

Figura 1. Escores (+ - desvio padrão) do teste TUG antes (PRE) e após (POS) o período de treinamento para grupo experimental (GE) e controle (GC).

\section{Avaliação de TINETTI}

$\mathrm{Na}$ comparação intragrupos e intergrupos do GC não houve diferença ( $p>0,05 ; E S=0,38)$ dos componentes relacionados ao equilíbrio após o período de treinamento, entretanto, observa-se que, no GE, houve aumento após o período de treinamento. A avaliação da marcha do teste de Tinetti no pré e pós período de intervenção demonstrou que houve melhora no GE $(p<0,05 ; E S=1,51)$. Nos resultados obtidos na avaliação do Tinetti somando-se os escores do equilíbrio e marcha, observou-se melhora $(\mathrm{p}<0,05 ; \mathrm{ES}=1,39)$ no GE que passou de 23,5 pontos para 25,5 pontos no pós intervenção, ou seja, de risco moderado a baixo risco de quedas. Os dados do teste de Tinetti encontram-se na Tabela 1.

\section{Parâmetros Morfológicos Musculares - Ultrassonografia}

Os grupos eram homogêneos antes do período de treinamento (PRE). O vasto lateral apresentou aumento médio de $3.6 \mathrm{~mm}$ do comprimento do fascículo após o treinamento para o GE, quando em estado de relaxamento 
$(\mathrm{p}<0,05 ; \mathrm{ES}=1,15)$. Durante a contração isométrica, observaram-se ganhos médios no comprimento do vasto lateral de $4,9 \mathrm{~mm}$ para o GE ( $\mathrm{p}<0.05$; $\mathrm{ES}=1,94)$, os quais não foram significativos para o $\mathrm{GC}(\mathrm{p}>0,05 ; \mathrm{ES}=0,02)$. A FIGURA 2 apresenta as alterações nas condições isométrica e relaxamento dos grupos GE e GC.

Tabela 1. Escores (+ - desvio padrão) da avaliação de Tinetti antes (PRE) e após (POS) o período de treinamento para grupo experimental (GE) e controle (GC).

\begin{tabular}{lcccccc}
\hline \multirow{2}{*}{$\begin{array}{l}\text { VARIÁVEL } \\
\text { (pontos) }\end{array}$} & \multicolumn{3}{c}{ GC } & \multicolumn{3}{c}{ GE } \\
\cline { 2 - 7 } & PRE & POS & $\Delta \%$ & PRE & POS & $\Delta \%$ \\
\hline TINETTI equilíbiro & $13,91 \pm 2,12$ & $13,82 \pm 1,78$ & 1 & $13,58 \pm 0,90$ & $14,08 \pm 1,24$ & 4 \\
TINETTI marcha & $10,45 \pm 1,44$ & $10,55 \pm 1,04$ & 1 & $9,92 \pm 1,31$ & $11,42 \pm 0,67^{a}$ & 15 \\
TINETTI total & $24,36 \pm 2,69$ & $24,36 \pm 1,86$ & 0 & $23,50 \pm 1,88$ & $25,50 \pm 1,00^{a}$ & 9 \\
\hline
\end{tabular}

a diferenças $(p<0,05)$ entre as condições (PRE e POS) no grupo experimental. $\beta$ diferenças $(p<0,05)$ entre os grupos (experimental e controle) na condição POS. $\Delta \%$ variação percentual

A

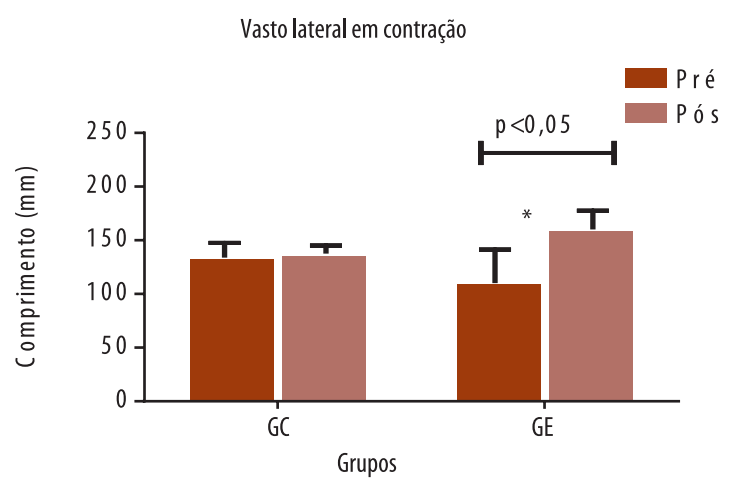

B

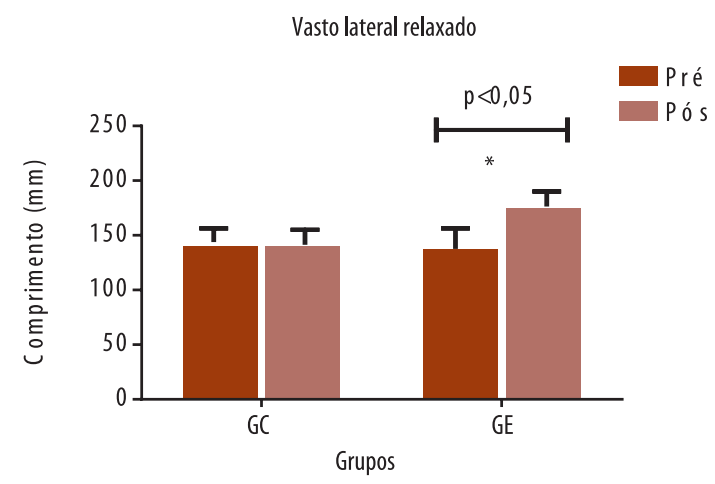

Figura 2. Comprimento do fascículo do músculo vasto lateral em contração isométrica (painel esquerdo - A) e em relaxamento (painel direito B).

O ângulo de penação do músculo vasto lateral, na condição relaxada, apresentou um aumento de $16 \%$ no GE $(\mathrm{p}<0.05$; ES=0,70) e uma redução de $1 \%$ no GC (p>0,05;ES=0,05). Durante a contração isométrica, observou-se um aumento de $12 \%$ no $\mathrm{GE}(\mathrm{p}<0,05 ; \mathrm{ES}=0,50)$, enquanto o grupo controle permaneceu inalterado ( $\mathrm{p}>0,05 ; \mathrm{ES}=0,01)$. A Figura 3 apresenta os resultados dos grupos experimentais antes (PRE) e após (POS) o treinamento.

Houve um aumento ( $\mathrm{p}<0,05 ; \mathrm{EF}=0,52)$ de $8 \%$ na espessura do músculo relaxado do grupo experimental. No grupo controle, houve uma redução $(\mathrm{p}>0,05 ; \mathrm{EF}=0,08)$ de $1 \%$ na mesma variável. Durante a contração isométrica, houve um aumento ( $\mathrm{p}<0,05 ; \mathrm{EF}=0,43)$ de $9 \%$ na espessura do músculo contraído do grupo experimental após o período de treinamento. O grupo controle não teve nenhuma variação percentual estatisticamente significativa ( $\mathrm{p}>0,05 ; \mathrm{EF}=0,03)$ entre a condição pré e pós. A figura 4 apresenta os resultados dos grupos experimentais antes (PRE) e após (POS) o treinamento. 


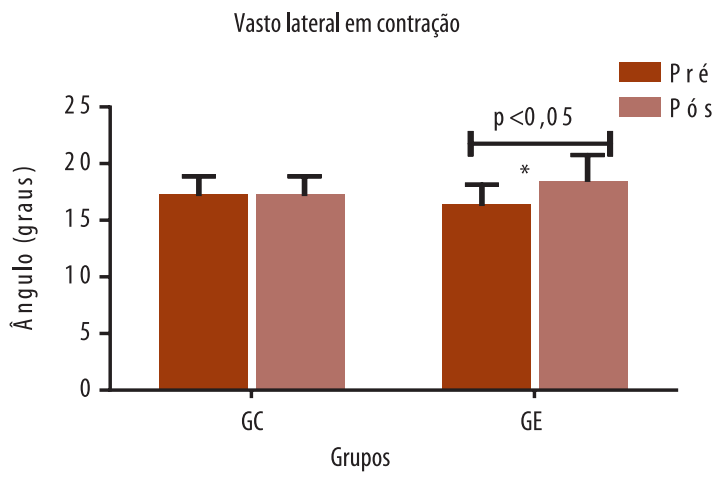

B

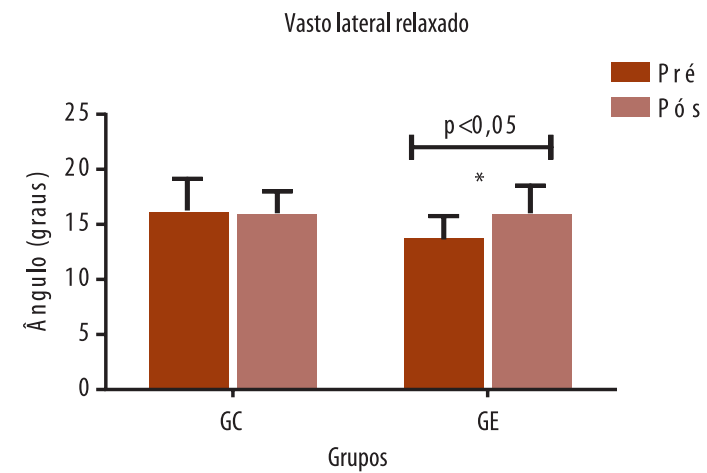

Figura 3. Comprimento médio (+- desvio padrão) do ângulo de penação do vasto lateral em contração isométrica (painel esquerdo -A) e em relaxamento (painel direito -B) dos grupos experimental (GE) e controle (GC) antes (PRE) e após (POS) o período de treinamento.

A

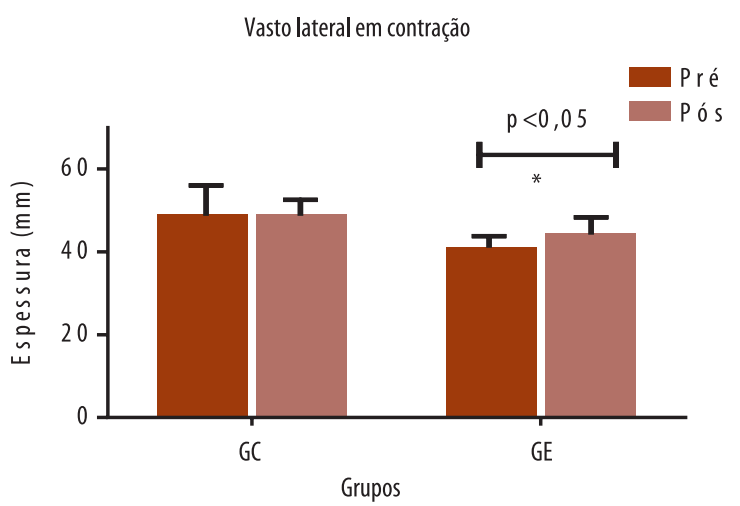

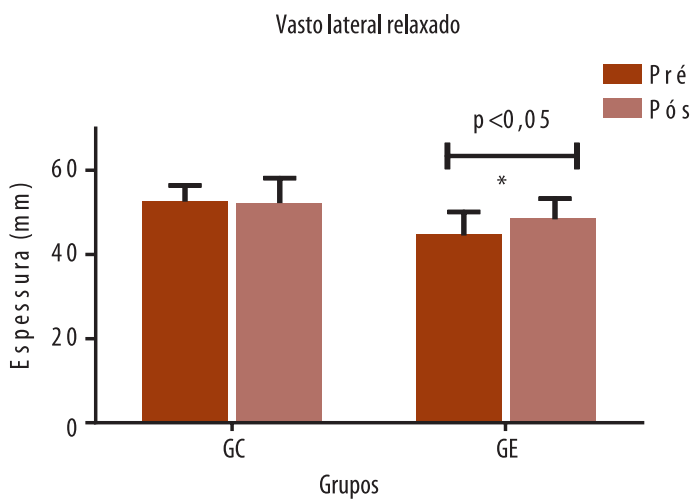

Figura 4. Comprimento médio (+- desvio padrão) da espessura muscular em contração isométrica (painel esquerdo -A) e em relaxamento (painel direito -B) dos grupos experimental (GE) e controle (GC) antes (PRE) e após (POS) o período de treinamento.

\section{DISCUSSÃO}

Os resultados do presente estudo mostraram que o treinamento isométrico com a utilização de posturas sustentadas do Método Isostretching promoveu adaptações na arquitetura muscular do músculo vasto lateral e melhorias na capacidade funcional e no equilíbrio em idosas.

O comprimento das fibras do músculo esquelético é um importante determinante das suas propriedades contráteis, fibras mais longas tendem a trabalhar em faixas de maior comprimento muscular e prover maior velocidade de encurtamento ${ }^{4,7,9,25}$.

Os resultados mostraram que após 12 semanas de treinamento com o método Isostretching, o comprimento do fascículo do vasto lateral no GE aumentou, em média, 27\% relaxado e $45 \%$ em contração isométrica submáxima. Os achados foram maiores do que os descritos por Reeves et al. ${ }^{26}$, que compararam os efeitos de duas modalidades de treinamento resistido durante 10 semanas, no excêntrico, o aumento foi de $20 \%$ e concêntrico, de $8 \%$ no comprimento do fascículo do vasto lateral de idosos. Tais diferenças podem ser atribuídas ao estresse mecânico e ao grau de estiramento imposto sobre o músculo ${ }^{26}$, os quais podem ter sido potencializados na maior tensão gerada nas contrações em diversos pontos do arco do movimento. De fato, 
é bastante conhecido que a tensão gerada numa contração isométrica é maior do que a concêntrica e excêntrica ${ }^{26}$.

O aumento do ângulo de penação de $16 \%$ com o músculo relaxado e $12 \%$ contraído, se assemelham aos achados de Blazevicz et al. ${ }^{27}$ que após 10 semanas de treinamento resistido em jovens, o aumento foi de 17,9 $\%$. Porém, menores aos encontrados por $\mathrm{Narici}^{26}, 35 \%$ de aumento no treinamento concêntrico e 5\% no excêntrico em idosos e de Suetta et al. ${ }^{28}$ $22 \%$ nos exercícios resistidos para o vasto lateral. Lee ${ }^{20}$ descreve em seu estudo um aumento de $4^{\circ}$ no ângulo de penação do gastrocnêmio medial após 10 semanas de trabalho isométrico por meio da eletroestimulação. O incremento no ângulo de penação determina adição de sarcômeros em paralelo pelo aumento da área de secção transversa fisiológica (PCSA) e pode ser considerado como uma estratégia de aumento da capacidade contrátil muscular ${ }^{26}$.

Alterações na espessura do vasto lateral foram observadas após o treinamento. Houve um aumento de $8 \%$ com o músculo relaxado e $9 \%$ em contração isométrica submáxima. Estes valores foram menores do que os encontrados por Reeves et al. ${ }^{26}$, que nas duas modalidades de treinamento obtiveram $12 \%$ de aumento. Sueta et al..$^{28}$, descrevem $14,8 \%$ de aumento no vasto lateral, após 12 semanas de treinamento resistidos em idosos, porém, nestes estudos, resistências adicionais foram utilizadas. Apesar dos nossos achados serem semelhantes aos de Seynnes e Narici ${ }^{29}, 7$ \% e Alegre et al. ${ }^{9}$, $6,9 \%$ de aumento da espessura do vasto lateral, estes resultados foram descritos em adultos jovens após treinamento de resistência leve a moderada.

A espessura é um dos parâmetros associados ao número de sarcômeros em paralelo, o que significa dizer que a maior espessura muscular representa maior capacidade de produção de força ${ }^{30}$. Embora a medição da espessura do músculo forneça uma indicação relativamente local de alterações no tamanho do músculo, tem mostrado ser um bom preditor de volume muscular do quadríceps ${ }^{26,27,29}$.

Nenhum estudo anterior avaliou os efeitos do método Isostretching sobre a arquitetura muscular. No entanto, a especificidade das adaptações musculares decorrentes do treinamento pelo isostretching é consistente com estudos anteriores que observaram os efeitos do treinamento isométrico em jovens e idosos ${ }^{11,18,19}$. Portanto, estes achados confirmam que programas que utilizam contações isométricas leves a moderadas podem prover importantes alterações nos parâmetros morfológicos musculares que influenciam a capacidade contrátil muscular.

Os déficits de força e potência muscular dificultam a capacidade de idosos em completar determinados movimentos funcionais que interferem nas atividades de vida diária. Melhorias na capacidade contrátil muscular têm sido apontadas como importantes no aprimoramento da capacidade funcional ${ }^{5,10,26}$.

Nos testes funcionais que dependem da capacidade contrátil, houve melhora de $11 \%$ no desempenho do teste de TUGT. No teste de Tinetti, nos itens relacionados à marcha, a melhora foi de $15 \%$ e equilíbrio $4 \%$. Resulta- 
dos semelhantes foram descritos com a utilização do mesmo protocolo de treinamento para idosos por Sanglard et al. ${ }^{21}$ e Carvalho e Assini ${ }^{22}$ quanto ao equilíbrio, marcha e capacidade funcional.

As melhoras significativas no desempenho dos testes funcionais, principalmente, pelo aumento da velocidade da marcha, demonstram uma maior agilidade no desenvolvimento da tarefa e podem ser atribuídos ao aumento da força muscular. As adaptações na arquitetura muscular observadas pelo aumento da espessura, ângulo de penação e comprimento do fascículo determinam melhora na geração de força e velocidade de contração.

Como limitação do estudo, a amostra apresentava elevado escore do IPAQ, e podem ter os resultados do estudo minimizados, uma vez que os efeitos de programas de atividade física tendem a ser maiores em sujeitos menos condicionados. Assim, as melhorias nos parâmetros morofológicos musculares, no equilíbrio e marcha poderiam ser mais expressivas em amostras compostas por sedentários e/ou fisicamente menos ativos.

\section{CONCLUSÃO}

O treinamento isométrico por meio de posturas sustentadas do Método Isostretching de intensidade leve a moderada, promoveu aumento do comprimento do fascículo, ângulo de penação e espessura muscular. Estas melhorias na capacidade contrátil podem ter sido determinantes no aprimoramento das capacidades funcionais ${ }^{26,29}$

\section{REFERÊNCIAS BIBLIOGRÁFICAS}

1. Ratanen T, Guralnik J, Sakari-Rantala R, Leveille S, Simonsick E, Ling S, Fried L. Disability, physical activity, and muscle strength in older women: the women's health and ageing study. Arch Phys Med Rehabil 1999;80:130-5.

2. Giné-Garriga M, Guerra M, Manini TM, Mari'-Dell'Olmo M, Pagès E, Unnithan V B. Measuring balance, lower extremity strength and gait in the elderly: Construct validation of an instrument. Arch Gerontol Geriatr 2010;51:199-204.

3. Katsiaras A, Newman AB, Kriska A, Brach J, Krishnaswami S, Feingold E, et al. Skeletal muscle fatigue, strength, and quality in the elderly: the Health ABC Study. J Appl Physiol 2005;99:210-6.

4. Aagaard P, Magnusson PS, Larsson B, Kjaer M, Krustrup P. Mechanical muscle function, morphology, and fiber type in lifelong trained elderly. Med Sci Sports Exerc 2007;39:1989-96.

5. Barry BK, Carson RG. The consequences of resistance training for movement control in older adults. J Gerontol A Biol Sci Med Sci 2004;59A(7)730-54.

6. Narici MV, Manganaris CN. Adaptability of ederly human muscles and tendons to increased loading. J Anat 2006;208:433-43.

7. Macaluso A, De Vito G. Muscle strenght power and adaptations to resistance training in older people. Eur J Appl Physiol 2004;91:450-72.

8. Narici MV, Manganaris CN. Plasticity of the Muscle-Tendon Complex With Disuse and Aging. Exerc Sports Sci Rev 2007;35(3):126-34.

9. Alegre LM, Jimènez F, Gonzalo-Orden M, Martin-Acero R, Aguardo X. Effects of dynamic resistance training on fascicle length and isometric strength. J Sports Sci 2006;24(5):501-8. 
10. Aagaard P, Andersen JL, Dyhre-Poulsen P, Leffers AM, Wagner A, Magnusson SP, et al. A mechanism for increased contractile strength of human pennate muscle in response to strength training: Changes in muscle architecture. J Physiol 2001;15:613-23.

11. Kanehisa H, Nagareda H, Kawakami Y, Akima H, Masani K, Kouzaki M. Effects of equivolume isometric training programs comprising medium or high resistance on muscle size and strength. Eur J Appl Physiol 2002;87:112-9.

12. Kubo K, Kanehisa H, Azuma K, Ishizu M, Kuno SY, Okada M, et al. Muscle architectural characteristics in women aged 20-79 years. Med Sci Sports Exerc 2003;35(1):39-44.

13. Steib S, Schoene D, Pfeifer K. Dose-Response Relationship of Resistance Training in Older Adults: A Meta-Analysis. Med Sci Sports Exerc 2010;42(5):902-14.

14. Hazzel T, Kenko K, Jakobi J. Functional benefit of power training for older adults. J Aging Physiol Act 2007;15(3):349-59

15. Orr R, Vos NJ, Singh NA, Ross DA, Stravinos TM, Fiatarone-Singh MA. Power training improves balance in healthy older adults. J Gerontol A Biol Sci Med Sci 2006;61A(1):78-85.

16. Pruitt LA, Taaffe DR, Marcus R. Effects of a one-year high-intensity versus low-intensity resistance training program on bone mineral density in older women. J Bone Miner Res 1995;10:1788-95.

17. Hortobagyi T, Tunnel D, Moody J, Beam S, DeVita P. Low- or high intensity strength training partially restores impaired quadriceps force accuracy and steadiness in aged adults. J Gerontol Biol Sci 2001;56A:B38-B47.

18. Kubiak RJ, Whitman KM, Johnston Rm. Changes in quadriceps femoris muscle strength using isometric exercise versus electrical stimulation. J Orthop Sports Phys Ther 1987;8(11):537-41.

19. Kubo K, Ikebukuro T, Yaeshimo K, Yata H, Tsunoda N, Kanehisa H. Effects of static and dynamic training on the stiffness on the blood volume of tendon in vivo. J Appl Physiol 2009;106:412-7.

20. Lee JW, Yoon SW, The effects of neuromuscular electrical stimulation on skeletal muscle architecture and qualitative proprierties in vivo. International journal of Contents 2009;5(4):35-9.

21. Sanglard RCF, Pereira JS, Henriques GRP, Gonçalves GB. A influência do isostretching nas alterações do equilíbrio em idosos. Rev Bras Ciênc Mov 2007;15(2):63-71.

22. Carvalho AR, Assini TCKA. Aprimoramento da capacidade funcional em idosos submetidos a uma intervenção por isostretching. Rev Bras Fisioter 2008;12(4):268-73.

23. Benedetti TRB, Mazo GZ, Barros MV. Aplicação do Questionário Internacional deAtividade Física para avaliação do nível de atividades físicas de mulheres idosas:validade concorrente e reprodutibilidade teste/reteste. Rev Bras Ciênc Mov 2004;12(1):25-33.

24. Bohannon R W. Reference values for the timed up and go test: a descriptive meta-analysis. J Geriatr Phys Ther 2006;29(2):64-8.

25. Mairet S, Maisseti O, Portero P. Homogeneity and reproducibility of in vivo fascicle length and penattion determined by ultrassonography in human vastus lateralis muscle. Science \& Sports 2006;21:268-72.

26. 26 Reeves ND, Maganaris CN, Longo S, Narici MV. Differential adaptations to eccentric versus conventional resistance training in older humans. Exp Physiol 2009;94(7):825-33.

27. Blazevich AJ, Cannavan D, Coleman DR, Horne S. Influence of concentric and eccentric resistance training on architectural adaptation in human quadriceps muscles. J Appl Physiol 2007;103:1565-75.

28. Suetta C, Andersen JL, Dalgas U, Berget J, Koskinen S, Aagaard P, et al. Resistance training induces qualitative changes in muscle morphology, muscle architecture, and muscle function in elderly postoperative patients. J Appl Physiol 2008;105:180-6. 
29. Seynnes OR, Boer M, Narici MV. Early skeletal muscle hypertrophy and architectural changes in response to high-intensity resistance training. J Appl Physiol 2007;102:368-73.

30. Degens H, Erskine RM, Morse CI. Disproportionate chages in skeletal muscle strength and size with resistance training and ageing. J Musculoskelet Neuronal Interact 2009; 106: 412-7.
Endereço para correspondência

Christina Cruz Cepeda

Universidade Positivo.

Rua Prof.Pedro Viriato Parigot de

Souza, 5.300

Campo Comprido

81280-330 - Curitiba, PR - Brasil

E-mail: christina.cepeda@up.com.br 